Results 19 studies were extracted, 37\% of which showed an increased incidence of diarrhoea within sub-groups, specifically in infants who were iron replete at baseline. 2 studies reported bloody diarrhoea. No clear relationship between type of intervention or volume of iron administered was observed.

Conclusion This review supports the current World Health Organisation (WHO) guidelines, which recommend the use of iron interventions only in areas with a high prevalence of anaemia. However, the potential unnecessary morbidity burden which may be induced by indiscriminate blanket population interventions must be reconsidered. Using the iron regulating hormone hepcidin, this review proposes a novel mechanism by which iron replete individuals may be at highest risk of diarrhoea as well as future methods of mitigating such adverse outcomes.

\section{G298(P) ZIKA VIRUS ASSOCIATED MENINGOENCEPHALITIS IN CHILDREN: ARE WE UNDER REPORTING?}

${ }^{1} \mathrm{G}$ Oligbu, ${ }^{2} \mathrm{~L}$ Pay, ${ }^{2} \mathrm{M}$ Fallaha, ${ }^{3} \mathrm{~L}$ Ahmed, ${ }^{4} \mathrm{OE}$ Oligbu, ${ }^{5} \mathrm{C}$ Durojaiye. ${ }^{1}$ Paediatric Infectious Diseases Research Group, Institute for Infection and Immunity, St George's, University of London, UK; ${ }^{2}$ Department of Paediatrics, Imperial College London, UK; ${ }^{3}$ Department of Paediatrics, Northwick Park Hospital, London, UK; ${ }^{4}$ Accident and Emergency Department, Queen Elizabeth Hospital, Woolwich, London, UK; ${ }^{5}$ Department of Infection and Tropical Medicine, Royal Hallamshire Hospital, Sheffield, UK

\subsection{6/archdischild-2018-rcpch.290}

Introduction Since 2015, an outbreak of Zika Virus infection $(\mathrm{ZiKV})$ has been reported in over 30 countries. Emerging evidence suggest $\mathrm{ZikV}$ causes a spectrum of neurologic diseases both directly and by secondary autoimmunity. In pregnancy, $\mathrm{ZiKV}$ is a well-known cause of congenital brain abnormalities, including microcephaly. ${ }^{1}$ It has also been linked with GuillainBarre syndrome. ${ }^{2}$ However, its association with meningoencephalitis is not well described, especially in children.

Methods We undertook a systematic review of the English literature published from 1947 to August 2017 to evaluate the risk factors, distribution, pathogenesis, clinical presentation, management and outcomes of encephalitis following Zika virus infection. Data sources included MEDLINE, EMBASE, Cochrane library, and references within identified articles. We also searched the papers using the ISI web of knowledge, to identify relevant articles and conference proceedings.

Results We identified 167 potential studies, of which 16 were duplicates and 140 were excluded on the basis of title and abstracts (figure 1). There were 9784 with suspected ZiKV in all age groups. 1411 cases were confirmed ZiKV either by RT-PCR in plasma, CSF and urine, ELIZA or MRI. There were $26(1.8 \%)$ cases of meningoencephalitis. Among eight studies reporting clinical characteristics and outcomes of 16 cases, the mean age was 43 years (Range 19-81) and $75 \%$ $(n=12)$ of cases were male. 63\% $(n=10)$ of the cases required admission to ITU, with a crude case fatality rate of $12 \%$ $(n=2)$.

Conclusions and clinical implications Complications from ZiKV, although uncommon, may be severe. With international spread, clinicians need to be aware that $\mathrm{ZiKV}$ may be associated with meningoencephalitis. Although our review found a paucity of evidence of Zika virus associated meningoencephalitis in children, standardising the collection and reporting for individual cases across regions and countries would further allow meaningful analysis of the data collected, enabling monitoring of trends over time.

\section{British Paediatric Neurology Association}

\section{G299 NUSINERSEN (SPINRAZA) IS THE FIRST DRUG APPROVED FOR SPINAL MUSCULAR ATROPHY (SMA): INITIAL EXPERIENCE IN PATIENTS WITH SMA TYPE 1 TREATED IN THE EXPANDED ACCESS PROGRAM (EAP)}

${ }^{1,2} \mathrm{M}$ Scoto, ${ }^{2} \mathrm{M}$ Main, ${ }^{2} \mathrm{P}$ Munot, ${ }^{3} \mathrm{~L}$ Edel, ${ }^{3} \mathrm{~F}$ Abel, ${ }^{3} \mathrm{E}$ Chan, ${ }^{2} \mathrm{~A}$ Manzur, ${ }^{1,2} \mathrm{~F}$ Muntoni.
${ }^{1}$ Dubowitz Neuromuscular Centre, Great Ormond Street, UCL Institute of Child, London, UK;
${ }^{2}$ Dubowitz Neuromuscular Unit, Great Ormond Street Hospital for Children, London, UK;
${ }^{3}$ Respiratory Unit, Great Ormond Street Hospital for Children, London, UK

10.1136/archdischild-2018-rcpch.291

Aim to report the experience in patients with SMA type 1 treated in the Nusinersen expanded access program (EAP) in a single centre that have the largest UK cohort of patients with SMA type 1 (24 patients). Infantile SMA type 1 is the most common form of classic proximal 5q-SMA and also the most severe with inability to achieve independent sitting and limited life expectancy. SMA is caused by mutations in the survival motor neuron 1 (SMN1) gene resulting in insufficient SMN protein levels. Nusinersen (commercial name SPINRAZA) is a modified antisense oligonucleotide that, administered via consecutive intrathecal injections, modulates the splicing of the SMN2 mRNA transcript to include exon 7, thereby increasing the production of full-length SMN protein. Following the promising results of a large international phase II clinical trial (ENDEAR) nusinersen has received FDA and EMA approval and is currently offered under EAP in several European countries.

Methods The motor abilities of all patients recruited in the EAP were systematically assessed using a validated score of motor function developed specifically for patients with SMA type 1 (CHOP-intend scale) and the Hammersmith Infant Neurological Examination-part 2 (HINE-2 motor milestone assessment). Information on SMN2 copies was collected from the patients" medical records while data on nutrition and respiratory function was collected at each visit prior drug administration.

Results Twenty-one/24 patients with SMA type 1 (age range 3 months- 9 years) were recruited into the EAP from March 2017. One patient is under evaluation while two patients are already recruited into an open label trial using the same drug. To date, patients in the EAP tolerated well the lumbar puncture procedure performed using local anaesthetic and no side effects or adverse events related to the drug were noted. In $75 \%$ of the patients an improvement of the CHOP-Intend total score was observed after the 4th or 5th injection.

Conclusion Our data confirm the safety of intrathecal Spinraza treatment in SMA type 1 as well as a degree of motor function improvement. More longitudinal data is necessary to evaluate the long-term benefits, the respiratory muscle response and the best responders. 